\title{
Diagnosis of snake envenomation using a simple phospholipase $A_{2}$ assay
}

\author{
Kalana Maduwage ${ }^{1,2}$, Margaret A. O'Leary ${ }^{3}$ \& Geoffrey K. Isbister ${ }^{1,3}$
}

\section{Received \\ 27 February 2014 \\ Accepted \\ 9 April 2014 \\ Published \\ 29 April 2014}

Correspondence and requests for materials should be addressed to G.K.I. Igeoff.isbister@ gmail.com)
'School of Medicine and Public Health, University of Newcastle, NSW 2308, Australia, ${ }^{2}$ Department of Biochemistry, Faculty of Medicine, University of Peradeniya, Sri Lanka, ${ }^{3}$ Department of Clinical Toxicology and Pharmacology, Calvary Mater Newcastle, Waratah, NSW 2298, Australia.

Diagnosis of snake envenomation is challenging but critical for deciding on antivenom use. Phospholipase $\mathrm{A}_{2}$ enzymes occur commonly in snake venoms and we hypothesized that phospholipase activity detected in human blood post-bite may be indicative of envenomation. Using a simple assay, potentially a bedside test, we detected high phospholipase activity in sera of patients with viper and elapid envenomation compared to minimal activity in non-envenomed patients.

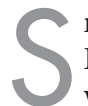
nake envenomation is considered a major medical and public health problem in the rural tropical world ${ }^{1}$. Each year there are 1 to 2 million cases of snake envenomation and potentially up to 100,000 deaths worldwide. Despite the burden of illness, the treatment of snake envenomation remains problematic. There is limited availability of antivenom in some countries, unacceptably high reaction rates to antivenom, and difficulties in diagnosing envenomation to allow early antivenom treatment in patients with definite envenomation ${ }^{2-4}$. A key issue for improving antivenom treatment in snake envenomation is to have a rapid and accurate test to determine if patients are envenomated and require antivenom ${ }^{5}$. Unfortunately the majority of clinical features and laboratory investigations indicative of envenomation only occur in established envenomation, where antivenom may be of limited or no benefit. It is therefore necessary to develop an early diagnostic test for envenomation that can be done at the bedside and is cheap and available in resource poor settings. Such tests have been developed for poisoning in the resource poor setting ${ }^{6,7}$.

Phospholipase $\mathrm{A}_{2}\left(\mathrm{PLA}_{2}\right)$ is a common toxin/component in snake venoms including in almost all elapid and viperid venoms ${ }^{8-10}$. Numerous studies have tested $\mathrm{PLA}_{2}$ activity in snake venoms and it is arguably a standard test when investigating venom activity. However, no studies have tested for $\mathrm{PLA}_{2}$ activity in the blood of patients with snake envenomation. Although $\mathrm{PLA}_{2}$ activity is unlikely to represent clinically important toxicity, it indicates that there is venom present in blood, and that there is likely to be systemic envenomation. We aimed to test the hypothesis that envenomated patients would have measureable PLA $\mathrm{A}_{2}$ activity in their blood compared to minimal activity in non-envenomated patients as the basis for a bedside test to diagnose snake envenomation.

\section{Results}

There were pre-antivenom samples for 32 patients with Russell's viper (Daboia russelii) envenomation, 35 with hump-nosed pit viper (Hypnale hypnale) envenomation, 3 with Indian cobra (Naja naja) envenomation, 2 with Indian krait (Bungarus caeruleus) envenomation, 5 with red-bellied black snake (Pseudechis porphyriacus) envenomation and 31 non-envenomated patients. PLA ${ }_{2}$ activity was detected in pre-antivenom sera in all patients with snake envenomation. The median $\mathrm{PLA}_{2}$ activity was $55.7 \mu \mathrm{mol} / \mathrm{ml} / \mathrm{min}$ (95\% percentiles: 18.0 to 226.2 ) for Russell's viper envenomation, $13.6 \mu \mathrm{mol} / \mathrm{ml} / \mathrm{min}$ (95\% percentiles: 9.7 to 24.5 ) for hump-nosed viper envenomation, $14.8 \mu \mathrm{mol} / \mathrm{ml} / \mathrm{min}$ (11.3 to 200.2 ) for cobra, $17.2 \mu \mathrm{mol} / \mathrm{ml} / \mathrm{min}$ (15.8 to 18.7$)$ for krait, and $98 \mu \mathrm{mol} / \mathrm{ml} /$ min (43 to 281) for black snake which were significantly different to non-envenomated patients (Median: $6.0 \mu \mathrm{mol} / \mathrm{ml} / \mathrm{min}$; $95 \%$ percentiles 2.3 to 8.4 ) [Fig. 1].

$\mathrm{PLA}_{2}$ activity was compared to venom specific enzyme immunoassays (EIA) for three of the snake venoms. There was good correlation between venom specific EIA and PLA 2 activity in the sera of patients with Russell's viper envenomation $(r=0.61 ; p=0.0002)$, hump-nosed pit viper envenomation $(r=0.49 ; p=0.003)$ and in multiple samples from the 5 black snake envenomations $(r=0.95 ; p<0.0001)$ [Fig. 2].

Venom specific EIA rapidly decreases after antivenom administration and the absence of detectable free venom indicates that sufficient antivenom has been administered ${ }^{11}$. A similar phenomenon occurred with $\mathrm{PLA}_{2}$ activity which decreased after antivenom administration and increased with apparent venom recurrence in Russell's viper 




Figure 1 Scatterplot of the phospholipase $A_{2}$ activity $(\mu \mathrm{mol} / \mathrm{ml} / \mathrm{min})$ for non-envenomated patients (green circles) versus patients with Russell's viper envenomation ( $D$. russelii; red circles), hump-nosed viper envenomation ( $H$. hypnale; blue circles), Indian cobra envenomation $(N$. naja; orange circles), Indian krait (B. caeruleus; purple circles) and redbellied black snake ( $P$. porphyriacus; black circles). The 6 groups had significantly different phospholipase $\mathrm{A}_{2}$ activities $(\mathrm{p}<0.0001$; KruskalWallis) and each snake envenomation group's phospholipase $\mathrm{A}_{2}$ activity was significantly different to non-envenomated samples by Dunn's multiple comparisons [shown with brackets; ${ }^{*}=\mathrm{p}<0.05, * *=$ $\mathrm{p}<0.0001]$.

envenomation and red-bellied black snake envenomation (Fig 3.). $\mathrm{PLA}_{2}$ appears to mirror the free venom concentrations and is another potential way to assess the efficacy of antivenom in vivo. Interestingly this differs to a previous study where antibodies to the phospholipase did not prevent hydrolysis of a different small chromogenic substrate $^{12}$.

\section{Discussion}

Currently no single laboratory investigation or bedside test exists to identify patients with systemic envenomation and the diagnosis involves a combination of clinical features and investigations ${ }^{3,11}$. This usually requires a level of medical knowledge to make the diagnosis and is difficult in resource poor settings. The 20 minute whole blood clotting test has been used as a simple bedside test to identify coagulopathy worldwide ${ }^{13,14}$. However, it has recently been shown to have a poor sensitivity in the clinical setting ${ }^{5}$ and is of no value for snakes that do not cause a coagulopathy such as kraits and cobras. The PLA 2 assay was positive in both coagulopathic snakes (Russell's viper and Hump-nosed viper) and neurotoxic snakes (kraits and cobras) in our sample of bites by Australian and Asian snakes. A simple bedside version of the $\mathrm{PLA}_{2}$ assay would therefore be a useful test in resource poor areas with limited access to medical care.

$\mathrm{PLA}_{2}$ activity in human sera will not determine the snake species responsible for envenomation, but will simply identify that the patient has systemic envenomation. The majority of snake antivenoms that are available are polyvalent so a test that simply indicates that the patient has snake envenomation is sufficient so that polyvalent antivenom can be administered.

The PLA 2 assay may also be particularly useful in neurotoxic envenomation to diagnose systemic envenomation early, before irreversible neurotoxicity develops. Most neurotoxicity is due to presy-

B

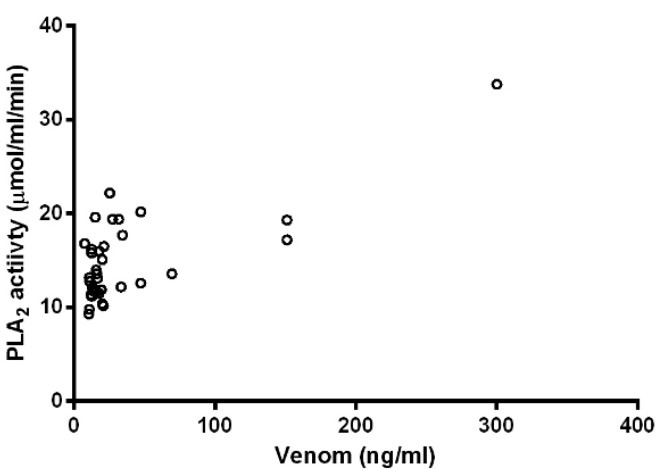

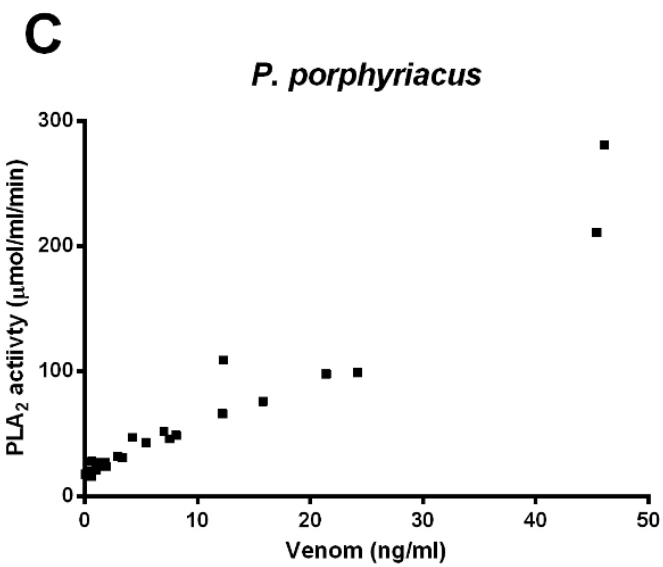

Figure $2 \mid$ Plots of phospholipase $A_{2}$ activity against venom concentration for samples from patients with envenomation from Russell's viper ( $D$. russelii; Panel A), hump-nosed viper (H. hypnale; Panel B) and red-bellied black snake (P. porphyriacus; Panel C). 

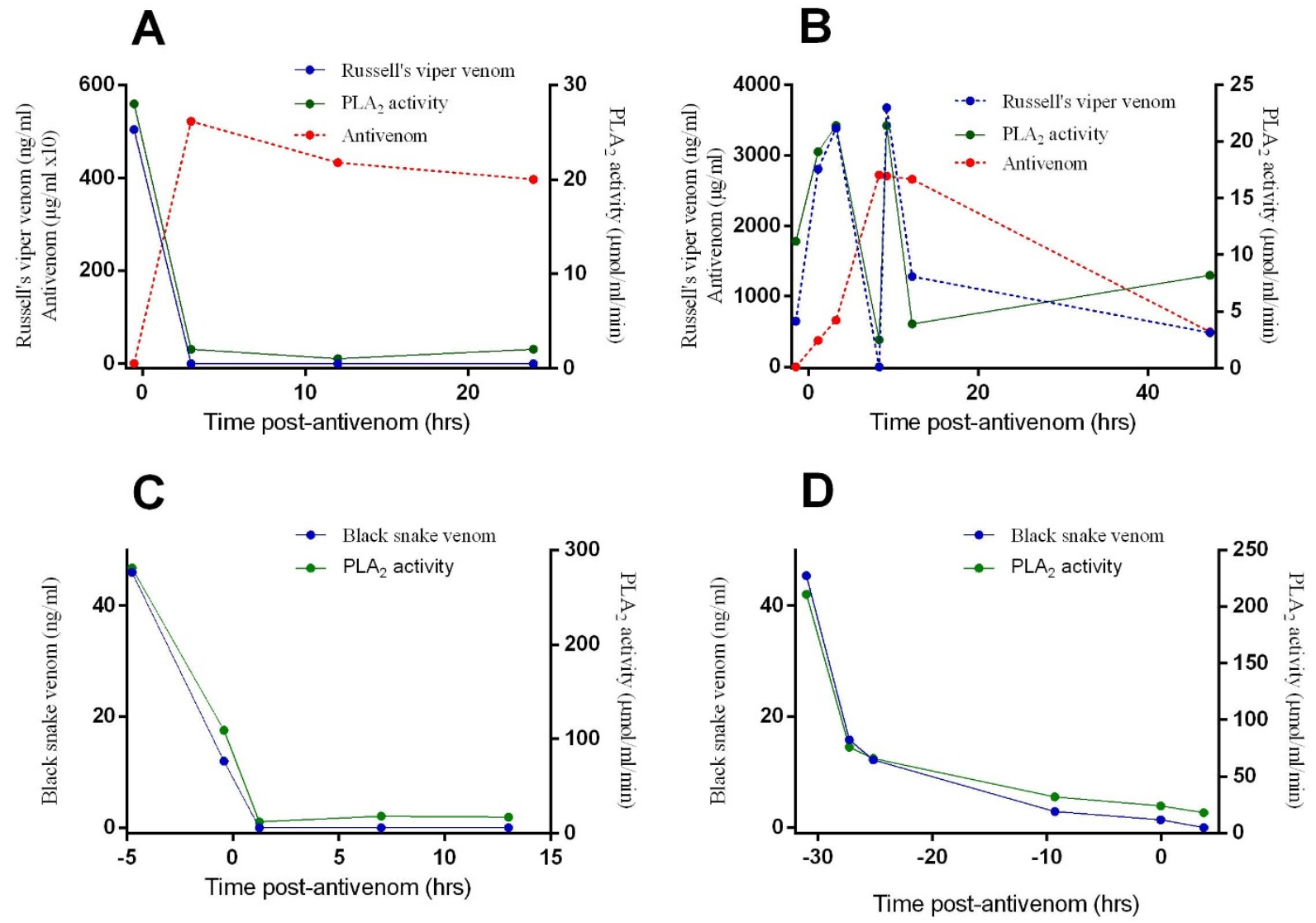

Figure 3 Plots of free venom concentration ( $\mathrm{ng} / \mathrm{ml}$; blue circles and lines), phospholipase $\mathrm{A}_{2}$ activity $(\mu \mathrm{mol} / \mathrm{ml} / \mathrm{min}$; green circles and lines) and antivenom concentrations ( $\mu \mathrm{g} / \mathrm{ml}$; red circles and dashed lines) versus time in a patient with Russell's viper envenomation where venom was undetectable post-antivenom (Panel A), a patient with Russell's viper envenomation where there was venom recurrence post-antivenom (Panel B), a patient with red-bellied black snake envenomation given early antivenom (Panel C) and a patient with red-bellied black snake envenomation given late antivenom (Panel D).

naptic neurotoxins (e.g. $\beta$-bungarotoxin in krait; Bungarus spp.), which cause irreversible nerve injury that can only be prevented and not reversed by antivenom. Once paralysis develops antivenom is ineffective. Venom is injected at the bite site and moves rapidly to the circulation. However, the onset of clinical neurotoxicity occurs over hours because it takes further time for the neurotoxins to distribute to the neuromuscular junctions and bind to the presynaptic membrane. It is therefore likely that $\mathrm{PLA}_{2}$ activity can be measured in patient serum within 30 to 60 minutes of the bite as venom enters the circulation. This would then allow patients with systemic envenomation to be identified rapidly and given antivenom prior to the development of neurotoxicity.

In this study we assessed the $\mathrm{PLA}_{2}$ activity of only a small number of snakes. In addition, we did not compare the increase in $\mathrm{PLA}_{2}$ in snake envenomation patients to patients with acute pancreatitis, sepsis and rheumatoid arthritis ${ }^{15,16}$, which may cause false positive results. This may not be a major problem because the test would be used in cohorts of patients bitten by snakes where the pre-test probability for snake envenomation is high. In contrast the test would be of much less value in cohorts of patients with suspected bites or clinical features where false positives would be more problematic. Larger cohorts of patients are required to determine if this is a major problem. There would be huge benefits for a $\mathrm{PLA}_{2}$ test because it would allow for fast, simple and easy identification of systemic envenomation, so that antivenom can be given. The $\mathrm{PLA}_{2}$ kit used in this study requires a microplate reader and is designed to run tests as batches making it expensive for testing when the patient presents. Several methods have been used to measure PLA 2 activity in serum samples ${ }^{12,17,18}$. It would therefore be feasible to develop a $\mathrm{PLA}_{2}$ assay that is inexpensive, allows individual tests to be done and can be done at the bedside or in a basic laboratory setting.

\section{Methods}

Patients with Russell's viper (Daboia russelii), hump-nosed pit viper (Hypnale hypnale), cobra (Naja naja) and krait (Bungarus caeruleus) envenomation were recruited prospectively from snakebites presenting to Chilaw Hospital (Western Province) and Polonnaruwa Hospital (North Central Province) in Sri Lanka between 2007 and 2011as part of prospective cohort studies and randomised controlled trials ${ }^{3}$. The studies were approved by the Ethical Review Committee, Faculty of Medicine, University of Colombo. Patients with no evidence of envenomation but who had been bitten by a snake were also recruited. In addition, cases of black snake (Pseudechis porphyriacus) were included from the Australian snakebite project $^{19}$. The design of the Australian snakebite project has previously been described in detail and approval has been obtained from the Hunter New England Area Health Human Research Ethics Committee and nineteen other Human Research and Ethics Committees covering all institutions involved around Australia ${ }^{19,20}$. Informed consent was obtained from all patients and the experiments were undertaken in accordance with the National Health and Medical Research Council guidelines.

Clinical data and serial serum and citrate samples were collected for all snakebites. Serum samples were tested with venom specific enzyme immunoassay (EIA) for Russell's viper venom and hump-nosed pit viper venom for Sri Lankan cases, and black snake venom for Australian cases, to confirm envenomation ${ }^{3,19}$. Clotting studies on citrate samples and clinical data were used to confirm systemic envenoming ${ }^{3,21,22}$. Non-envenomed cases were defined as patients with a suspected snakebite where no venom was detected in serum samples, clotting studies were normal and the patient remained asymptomatic.

Serum samples were analysed for $\mathrm{PLA}_{2}$ activity by Cayman $\mathrm{SPLA}_{2}$ assay kit (\#765001Cayman Chemical Company, USA) according to manufacturer instructions. We compared the PLA $\mathrm{P}_{2}$ activity of Russell's viper, hump-nosed pit viper and black snake envenomed patients with non-envenomed patients. Furthermore, we investigated the correlation between PLA $\mathrm{A}_{2}$ activity and venom specific EIA 
concentration in three different snakes. In selected patients we measured serial serum samples for $\mathrm{PLA}_{2}$ activity, including before and after antivenom.

The PLA $A_{2}$ activities of patient sera from different snake groups and the nonenvenomated snake bites were compared with the non-parametric Kruskal-Wallis test and multiple comparisons with Dunn's test. Correlation between $\mathrm{PLA}_{2}$ activity and venom concentrations was compared with Spearman's test. Data is presented as medians and interquartile ranges or $95 \%$ percentiles, and ranges.

1. Kasturiratne, A. et al. The global burden of snakebite: a literature analysis and modelling based on regional estimates of envenoming and deaths. PLoS.Med. 5, e218 (2008).

2. Lalloo, D. G., Theakston, R. D. \& Warrell, D. A. The African challenge. Lancet 359, 1527 (2002)

3. Isbister, G. K. et al. A randomised controlled trial of two infusion rates to decrease reactions to antivenom. PLoS One 7, e38739 (2012).

4. Ireland, G. et al. Changes in serial laboratory test results in snakebite patients: when can we safely exclude envenoming? Med J Aust 193, 285-290 (2010)

5. Isbister, G. K. et al. Diagnostic 20-min whole blood clotting test in Russell's viper envenoming delays antivenom administration. QJM (2013).

6. Shihana, F., Dissanayake, D., Dargan, P. \& Dawson, A. A modified low-cost colorimetric method for paracetamol (acetaminophen) measurement in plasma. Clin Toxicol (Phila) 48, 42-46 (2010).

7. Shihana, F., Dissanayake, D. M., Buckley, N. A. \& Dawson, A. H. A simple quantitative bedside test to determine methemoglobin. Ann Emerg Med 55, 184-189 (2010).

8. Birrell, G. W. et al. The diversity of bioactive proteins in Australian snake venoms. Mol Cell Proteomics 6, 973-986 (2007).

9. Mukherjee, A. K. A major phospholipase A from Daboia russelii russelii venom shows potent anticoagulant action via thrombin inhibition and binding with plasma phospholipids. Biochimie (2013).

10. Kang, T. S. et al. Enzymatic toxins from snake venom: structural characterization and mechanism of catalysis. FEBS J 278, 4544-4576 (2011).

11. Isbister, G. K. et al. Snakebite in Australia: a practical approach to diagnosis and treatment. Med J Aust 199, 763-768 (2013).

12. Petrovic, N., Grove, C., Langton, P. E., Misso, N. L. \& Thompson, P. J. A simple assay for a human serum phospholipase A2 that is associated with high-density lipoproteins. J Lipid Res 42, 1706-1713 (2001).

13. Warrell, D. A. et al. Poisoning by bites of the saw-scaled or carpet viper (Echis carinatus) in Nigeria. Quarterly Journal of Medicine 46, 33-62 (1977).

14. Sano-Martins, I. S. et al. Reliability of the simple 20 minute whole blood clotting test (WBCT20) as an indicator of low plasma fibrinogen concentration in patients envenomed by Bothrops snakes. Butantan Institute Antivenom Study Group. Toxicon 32, 1045-1050 (1994).
15. Kortesuo, P. T., Nevalainen, T. J., Buchler, M. \& Uhl, W. Characterization of two phospholipases A2 in serum of patients with sepsis and acute pancreatitis. Eur $J$ Clin Chem Clin Biochem 30, 263-269 (1992).

16. Green, J. A. et al. Circulating phospholipase A2 activity associated with sepsis and septic shock is indistinguishable from that associated with rheumatoid arthritis. Inflammation 15, 355-367 (1991).

17. Tan, N. H. \& Tan, C. S. Acidimetric assay for phospholipase A using egg yolk suspension as substrate. Anal Biochem 170, 282-288 (1988).

18. Price, J. A. 3rd. A colorimetric assay for measuring phospholipase A2 degradation of phosphatidylcholine at physiological pH.J Biochem Biophys Methods 70, 441-444 (2007).

19. Churchman, A. et al. Clinical effects of red-bellied black snake (Pseudechis porphyriacus) envenoming and correlation with venom concentrations: Australian Snakebite Project (ASP-11). Med J Aust 193, 696-700 (2010).

20. Isbister, G. K., Brown, S. G., MacDonald, E., White, J. \& Currie, B. J. Current use of Australian snake antivenoms and frequency of immediate-type hypersensitivity reactions and anaphylaxis. Med J Aust 188, 473-476 (2008).

21. Isbister, G. K. et al. Factor deficiencies in venom-induced consumption coagulopathy resulting from Australian elapid envenomation: Australian Snakebite Project (ASP-10). J Thromb Haemost 8, 2504-2513 (2010).

22. Maduwage, K. et al. Hump-nosed pit viper (Hypnale hypnale) envenoming causes mild coagulopathy with incomplete clotting factor consumption. Clin Toxicol (Phila) 51, 527-531 (2013).

\section{Author Contributions}

K.M. conducted the laboratory experiments, analysed the data and drafted the manuscript; M.A.O. assisted with the laboratory experiments; G.K.I. designed the study, assisted in data analysis and edited the manuscript.

\section{Additional information}

Competing financial interests: The authors declare no competing financial interests.

How to cite this article: Maduwage, K., O’Leary, M.A. \& Isbister, G.K. Diagnosis of snake envenomation using a simple phospholipase $\mathrm{A}_{2}$ assay. Sci. Rep. 4, 4827; DOI:10.1038/ srep04827 (2014).

This work is licensed under a Creative Commons Attribution-NonCommercialNoDerivs 3.0 Unported License. The images in this article are included in the article's Creative Commons license, unless indicated otherwise in the image credit; if the image is not included under the Creative Commons license, users will need to obtain permission from the license holder in order to reproduce the image. To view a copy of this license, visit http://creativecommons.org/licenses/by-nc-nd/3.0/ 